\author{
G.0. Abel1 \\ University of California \\ Harold G. Corwin, Jr. \\ University of Texas, Austin
}

We are searching for clusters of galaxies on photographs taken for the Southern Sky Survey with the U.K. Schmidt telescope at Siding Spring, and we are preparing a comprehensive catalogue of the southern clusters. The purpose is to extend the early cluster survey made from the Palomar Sky Survey (Abell 1958) to full-sky coverage. We are taking special pains to delineate those clusters in the new southern survey that as nearly as possible match those criteria set for the northern survey clusters to be included in a homogeneous statistical sample. It is our expectation, therefore, that there will be a homogeneous all-sky sample of clusters for studying the large-scale distribution of matter in the universe.

The new southern cluster survey is now approximately half complete. We have also revised and updated the northern cluster catalogue, and we plan to publish both together.

THE REVISED NORTHERN CATALOGUE

The revised cluster catalogue, assembled from the Palomar Survey, contains many corrections that we have found or that have been called to our attention (the vast majority being slight adjustments to the equatorial coordinates), and some additional information. The following are tabulated for each cluster:

1. Catalogue number.

2. Equatorial coordinates to $\pm 1^{\prime}$ for the equinox of 1950 .

3. Precession in right ascension and declination.

4. Galactic coordinates on the modern system.

5. The photored magnitude, $\mathrm{R}_{10}$, of the tenth brightest galaxy.

6. Distance class.

7. Richness class.

8. Raw counts of galaxies within the Abell radius, corrected for the field.

9. Redshifts, when available.

The catalogue numbers of the clusters will remain the same, inasmuch as these numbers are in rather wide use. As a consequence, because the coordinates have been precessed, the clusters will no longer be numbered precisely in order of increasing right ascension.

G. O. Abell and G. Chincarini (eds.), Early Evolution of the Universe and Its Present Structure, 179-184.

(C) 1983 by the IAU. 
The identification of clusters that meet the criteria for inclusion in the homogeneous sample remains as before, and clusters that do not meet the criteria are indicated with an asterisk. The criteria are: 1) the cluster must be in a region clear enough of the Milky Way to assure reasonably complete identification. The galactic latitude limits vary somewhat with longitude (see Abel1 1958); 2) the redshift, estimated from $R_{10}$, must 1 ie in the range $0.02 \leq z \leq 0.2 ; 3$ ) there must be 50 or more galaxies within the Abell radius that are brighter than $\mathrm{R}_{3}+2$, after correction for the background.

\section{THE SOUTHERN CLUSTER CATALOGUE} will be given:

For each cluster in the southern catalogue the following data

1. Catalogue number, in order of increasing right ascension, beginning with 2713 .

2. Equatorial coordinates (to $\pm 1^{\prime}$ ), for the equinox 1950 .

3. Precession in right ascension and declination.

4. Galactic coordinates on the modern system.

5. Plate coordinates, in millimeters, measured from the southeast corner.

6. Bautz-Morgan class.

7. Abell class (regular or irregular).

8. Visual magnitudes, $\mathrm{V}_{1}, \mathrm{~V}_{3}$, and $\mathrm{V}_{10}$, of the first, third, and tenth galaxies.

9. Raw counts of galaxies within the Abell radius, corrected for the field.

10. Distance class.

11. Richness class.

12. Redshift, when available.

As in the northern catalogue, many clusters not meeting the criteria for the homogeneous sample will be included. Because of the far higher quality of the U.K. Schmidt survey plates, many more of such clusters are being catalogued than in the northern survey; most of these are clusters of estimated $z>0.2$. Also 1 isted in the southern catalogue, but marked as not in the homogeneous sample, are clusters in the fields that overlap the northern survey but which were missed by Abell (1958). A few of these do meet the criteria for the sample (see below), but are nevertheless marked with an asterisk in order not to contaminate any statistical differences that may exist between the completeness levels of the northern and southern catalogues.

\section{PROCEDURES FOR THE SOUTHERN CLUSTER SURVEY}

Experience since the northern catalogue was completed has taught us to include some additional data, for example, the morphological classes of clusters and the estimated magnitudes of the first and third brightest galaxies, in addition to the tenth. There are, however, four significant differences between the procedures used in the northern and southern catalogues: 1) the color sensitivity of the plates used for the survey; 2) the use of both glass originals and film copies; 3 ) the use of visual rather than photored magnitudes; and 4) the manner of correction for field galaxies. 


\section{BLUE VERSUS RED PLATES}

In the northern survey all clusters were identified and measured on the red (103a-E) plates of the Palomar Sky Survey. Because of their redshifts, even clusters of moderate distance $(z \sim 0.2)$ are far more conspicuous on the red (E) than on the blue (0) emulsion. In the southern survey, however, all data are being taken from the blue (IIIa-J) Siding Spring plates rather than from the red plates taken with the ESO Schmidt in Chile. There are three reasons: 1) the U.K. blue survey is now nearly complete, and its use saves us an estimated two years; 2) the southern survey fields are only 5 degrees across, because the field of view of the ESO Schmidt can cover only that area with a modest overlap. The U.K. Schmidt, however, like the Palomar Schmidt, has a field of 6.6 degrees, which provides a very large overlap between adjacent fields, giving us a splendid opportunity to check thoroughly our completeness and consistency; and 3) despite the redshifts of remote clusters, the U.K. J plates reveal more faint clusters and with higher resolution than do the ESO red plates. The ESO exposures are not as full as would be desirable, we understand because of telescope flexures.

In fact, the U.K Schmidt J plates show more distant and fainter clusters, and with better resolution, than do the early 103a-E (red) plates of the Palomar survey, despite the color advantage of the latter. The U.K. Schmidt is an improved version of the Palomar instrument, with less flexure, it has automatic guiding (not available in the 1950s, when the Palomar survey was completed), and the $\mathrm{J}$ emulsion has substantially higher resolution than the $103 \mathrm{a}-\mathrm{E}$ emulsion. Thus, although we are using blue rather than red plates, we are obtaining far better results than was possible with the earlier Palomar survey.

\section{PLATES VERSUS FILM COPIES}

All data for the northern cluster were taken from original glass plates of the Palomar Sky Survey. On the other hand, we have utilized both original U.K. Schmidt plates and film copies in our southern survey. The plates are either those taken for the Southern Survey that had been rejected for the purpose of reproduction, but for reasons that do not affect their value for our work, or plates centered on Southern Survey fields, taken under identical conditions of exposure and processing, but for non-survey purposes. Most of these plates are on file at the Royal Observatory Edinburgh, and all of those available (nearly half the total southern sky) have been searched for clusters (the overwhelming majority by HGC).

The survey of the remaining fields is being done on the film copies, mostly by GOA. Such would not have been possible with the Palomar Survey; the paper print copies were prepared to maintain the limiting magnitude of the originals, but at the expense of greatly increased contrast, making recognition of many distant clusters, clearly visible on the original plates, impossible on the prints. Even the glass negative copies of the Palomar Survey have contrasts too great to be optimum for a cluster survey.

On the other hand, the film copies of the U.K. Schmidt Survey plates are generally of excellent quality. Our experience has shown that for our purposes the copies are nearly indistinguishable in quality 
from the original plates. Fortunately, the large overlap between adjacent fields makes it possible for us to verify that no loss in completeness or precision results from use of the film copies.

\section{MAGNITUDES}

A11 our magnitude estimates are made with step scales consisting of galaxy images assembled from cut-outs of images on film copies of U.K. Schmidt photographs. The step-scale images are calibrated by comparing them with images of galaxies of known magnitude on the survey plates, by exactly the same procedure with which we compare the stepscale images with galaxies in our identified clusters. The magnitudes of galaxies serving as standards come from a variety of sources, including photoelectric measures by one of us (HGC) in South Africa. Because we record our step-scale values, we can refine our calibration at any time.

What needs to be explained is how we reconcile photored magnitudes in the northern survey and visual magnitudes in the south, estimated from blue plates. The brightest galaxies in most rich clusters are ellipticals and early spirals, which have $B-V$ colors in the range 0.7 to 1.0 -- roughly all the same to the precision of our step-scale estimates. Consequently, we assume that visual magnitudes can be estimated on blue plates, and that we can give appropriate transformation equations to relate blue, visual, or red magnitudes. Fortunately, we can check our assumptions with the substantial overlap data in fields also included in the northern survey. We have taken care to account for the differences in the $\mathrm{K}$-corrections in different magnitude systems in our calibration.

\section{FIELD CORRECTIONS}

When the northern survey was carried out there was no objective knowledge of the apparent luminosity function (distribution of galaxies with apparent magnitude). Consequently, corrections for the background and foreground field were made by counting galaxies both in the clusters and in several regions of the same area and in the same magnitude range in the surrounding field; the latter were averaged and subtracted from the former.

Because of superclustering, field counts so obtained are quite unreliable, and we suspect this to be a serious source of error in the homogeneity of the northern cluster catalogue. Now we can avoid the difficulty; there exist extensive counts of galaxies as a function of apparent magnitude. Those counts in the magnitude range relevant to our work are by Brown (1979) and by Rainey (1977), and they are in excellent agreement with each other (Abe11 1978). Here we use Rainey's counts, which are complete to $\mathrm{V}=19.5$ in three widely separated directions in the sky. We.simply subtract from our count of galaxies within the Abell radius and prescribed magnitude interval in a cluster the number of galaxies Rainey's observations indicate should be due to the average field. 


\section{CONSISTENCY OF NORTH AND SOUTH SURVEYS}

There are 135 clusters in common with the northern survey and partially completed southern survey. For these, the right ascensions measured on the northern and southern survey plates, $\alpha_{N}$ and $\alpha_{S}$, respectively, and the declinations $\delta_{N}$ and $\delta_{S}$ have the following mean differences:

$$
\begin{aligned}
& <\alpha_{N}-\alpha_{S}>=0.04 ; \quad \sigma=0.16 ; \\
& <\delta_{N}-\delta_{S}>=0 ! 54 ; \sigma=1 ! 92 .
\end{aligned}
$$

The mean ratio of raw counts of galaxies in the southern to the northern clusters, after correction for the field, is:

$$
<\mathrm{N}\left[\leq\left(V_{3}+2\right)\right] / \mathrm{N}\left[\leq\left(R_{3}+2\right)\right]>=1.17 ; \sigma=0.49 \text {. }
$$

We expected a large difference in the counts of galaxies in clusters, not only because of the sensitivity of the counts to small errors in the step-scale magnitude estimates, but because of the effect of superclustering on the background correction mentioned above. We are gratified that the agreement between the richnesses of the clusters as observed in the north and south is as good as it is.

The mean magnitude difference between $V_{10}$ in the south and $R_{10}$ in the north is:

$$
<\mathrm{V}_{10}-\mathrm{R}_{10}>=0.63 \mathrm{mag} ; \sigma=0.83 \text {. }
$$

The scatter is large, as expected for step-scale magnitude estimates. Nevertheless, the mean difference between the visual and red magnitudes is almost precisely the expected value for galaxies with $B-V$ colors of 1.0. We believe that most of the large scatter is due to the estimates of magnitudes in the north survey. Twenty-five years more experience, far superior plate quality, better calibration, and a much higher altitude above the horizon lead us to expect the magnitude estimates in the south to be more reliable. (The zone of overlap between the two surveys is low in the Palomar sky, where atmospheric extinction and seeing are at their worst.)

of the 135 clusters in common:

17 were classed richness " 0 " in both north and south;

19 were classed richness " 0 " in the north but not the south;

16 were classed richness " 0 " in the south but not the north. The mean difference between the richness classes $r(N)$ and $r(S)$ in the north and south, respectively, is:

$$
<\mathrm{r}(\mathrm{N})-\mathrm{r}(\mathrm{S})>=-0.19 \text { class; } \sigma=0.87 \text { class. }
$$

Abell (1958) had originally intended the richness classes to be two standard deviations wide. He was evidently slightly too optimistic, at least for the southernmost clusters in the northern survey. 


\section{COMPLETENESS}

Among the clusters in the overlap region, in the south nine were missed in the first screening (before consulting the Abell catalogue); only three would make the statistical sample. However, 66 clusters were picked up in the south that are richer than $\mathrm{N}\left[\leq\left(\mathrm{V}_{3}+2\right)\right]$ $=50$. Of these, 17 have $V_{10} \leq 18.5$ (the 1imit corresponds to $\mathrm{R}_{10}=17.9$ after correction for extinction, the red magnitude corresponding to $z=0.2)$.

Thus, the 135 clusters found in the north survey should have been 152 , suggesting an incompleteness of $11 \%$ in the northern catalogue at declinations south of -18 degrees.

\section{REFERENCES}

Abe11, G.0. 1958, Ap. J. Suppl., 3, 211 .

Abe11, G.0. 1978, The Large-Scale Structure of the Universe, ed.

M. Longair and J. Einasto (Dordrecht: Reide1), p. 253.

Brown, G.S. 1979 , A. J., 84, 1647.

Rainey, G.W. 1977, Ph.D. Thesis, University of California, Los Angeles.

\section{DISCUSSION}

I.E. Segal: Isn't it the case that your concept of cluster is inherently distance-dependent (and hence presumably redshift-dependent)? That is, requirement associated with a fixed magnitude difference implies different physical diameters for clusters at different distances, indeed a rather systematic trend with distance for physical diameters, does it not?

Abezl: The identification of clusters depends on their recognizability; we have no a priori information on redshift, magnitudes or distances. The data given for each cluster are operationally defined and are independent of cosmological assumptions. (Although the counting radius, defined in terms of redshift estimated from $\mathrm{V}_{10}$, is a constant linear distance only for conventional cosmologies.) Of course, the interpretation of the catalogued data is up to the cosmologist who uses the catalogue.

Thompson: In the revised version of the northern cluster survey, will the Coma cluster be changed to richness class 3 ? Such a change is important because too many astronomers presume that Coma is an average rich cluster, while, in fact, it is uncommonly rich.

Abezl: You are right. I am inclined, for consistency with original counts, to put this sort of correction under "comments." 